\title{
Development of Lignin-based Polycarboxylates as a Plasticizer for Cement Paste via Peracetic Acid Oxidation
}

Jong-Chan Kim, ${ }^{\mathrm{a}}$ June-Ho Choi, ${ }^{\mathrm{b}}$ Jong-Hwa Kim, ${ }^{\mathrm{b}}$ Seong-Min Cho, ${ }_{\mathrm{b}}$ Sang-Woo Park, ${ }^{\mathrm{a}}$ Young-Min Cho, ${ }^{\mathrm{a}}$ Se-Yeong Park, ${ }^{\mathrm{c}}$ Hyo Won Kwak, ${ }^{\text {a,d }}$ and In-Gyu Choi ${ }^{\mathrm{a}, \mathrm{d} *}$

Kraft lignin $(\mathrm{KL})$ was oxidized by peracetic acid, which is generated by mixing acetic acid and hydrogen peroxide, to produce polycarboxylates for use as a plasticizer for cement paste. Peracetic acid cleaves the aromatic ring structure of $\mathrm{KL}$ and introduces carboxylate groups with ring-opened chain structure. After oxidation, the water-soluble fraction (Cx-lig) was obtained, and the performance of the $\mathrm{Cx}$-lig as a plasticizer was compared with two commercial plasticizers, lignosulfonate (LS) and polycarboxylate ether (PCE). In mortar table tests, the increase in cement fluidity with the Cx-lig was greater than with LS and PCE. Fourier-transform infrared spectroscopy, carbon-13 nuclear magnetic resonance, gel permeation chromatography, elemental analysis, and charge density analysis were used to determine the structure of the Cx-lig. Considering all the results, the Cx-lig had a polycarboxylate structure containing numerous carboxylate groups, and their high charge density was the key factor that caused the Cx-lig to increase the cement fluidity more than LS or PCE.

Keywords: Kraft lignin modification; Peracetic acid oxidation; Polycarboxylates; Plasticizer; Charge density

Contact information: a: Department of Agriculture, Forestry, and Bioresources, College of Agriculture and Life Science, Seoul National University, Seoul 08826, Republic of Korea; b: Department of Forest Sciences, College of Agriculture and Life Sciences, Seoul National University, Seoul 08826, Republic of Korea; c: Department of Forest Biomaterials Engineering, College of Forest and Environmental Sciences, Kangwon National University, Chuncheon 24341, Republic of Korea; $d$ : Research Institute of Agriculture and Life Sciences, College of Agriculture and life Sciences, Seoul National University, Seoul 08826,

Republic of Korea; * Corresponding author: cingyu@ snu.ac.kr

\section{INTRODUCTION}

Lignin, one of the major components of woody biomass, is an aromatic and heterogeneous polymer. This material is mainly composed of three types of monolignols: p-coumaryl, coniferyl, and sinapyl alcohols. During various treatments and pulping processes, such as organosolv pretreatment and kraft pulping, a great amount of lignin is produced as a by-product, called technical lignin. Based on strategies for total utilization of woody biomass, many researchers have made notable efforts to improve the processes' economic efficiency and use these by-products by modifying the lignin structure and attaching a variety of functional groups such as carboxylate groups (Figueiredo et al. 2017; Sun et al. 2017), epoxide groups (El Mansouri et al. 2011; Ferdosian et al. 2012), and sulfonate groups (Aro and Fatehi 2017; Gao et al. 2019). Consequently, modified lignin has been used in various forms, such as monomers of polymers, bioplastic composites, adsorbents, and cement additives (Ouyang et al. 2009; He and Fatehi 2015; Zheng et al. 2019). 
Kraft lignin (KL) composes approximately $85 \%$ of lignin production and is a byproduct of kraft pulping. The kraft process produces pulp with the usage of sodium sulfide and sodium hydroxide. During kraft pulping, hydrosulfide and hydroxide anions not only decompose the lignin but also change its molecular structure, including the addition of thiol groups (Chakar and Ragauskas 2004). Consequently, $\beta$-aryl ether linkages decrease, and $\mathrm{C}-\mathrm{C}$ bonds among aromatic and aliphatic structures increase. Due to the cleavages of $\beta$ aryl ether linkages, phenolic hydroxyl groups on the lignin structure increase. Moreover, these C-C bonds, which are not easily cleaved without a metallic catalyst (Zhao et al. 2018), can prevent derivatives of KL from degrading into small molecules. This behavior is due to the sulfur in KL, which can easily deactivate a metal catalyst (Narani et al. 2015). These $\mathrm{C}-\mathrm{C}$ bonds maintain the chain structure after chemical reactions because they are too strong to be cleaved (Luo 2012).

Carboxylation is a reaction that introduces carboxylate groups onto the chemical structure. Specifically, this reaction is beneficial to making negatively charged materials for use as polymers (Lee and Park 2000) or cement additives. However, it is necessary to use suitable catalysts such as (2,2,6,6-tetramethylpiperidin-1-yl)oxyl (TEMPO), cobalt, or rhodium to perform carboxylation. Many studies have been conducted to introduce carboxylate groups onto the chemical structure. It is better to use less expensive chemicals to attach carboxylate groups, rather than very expensive catalysts. Many studies have examined chemical modification with peracetic acid, which has been used as a powerful oxidizing agent that can react even at room temperature. For example, the mechanisms of hydroxylation and carboxylation for KL by peracetic acid have been demonstrated in previous research (Fig. 1) (Barros et al. 2010).
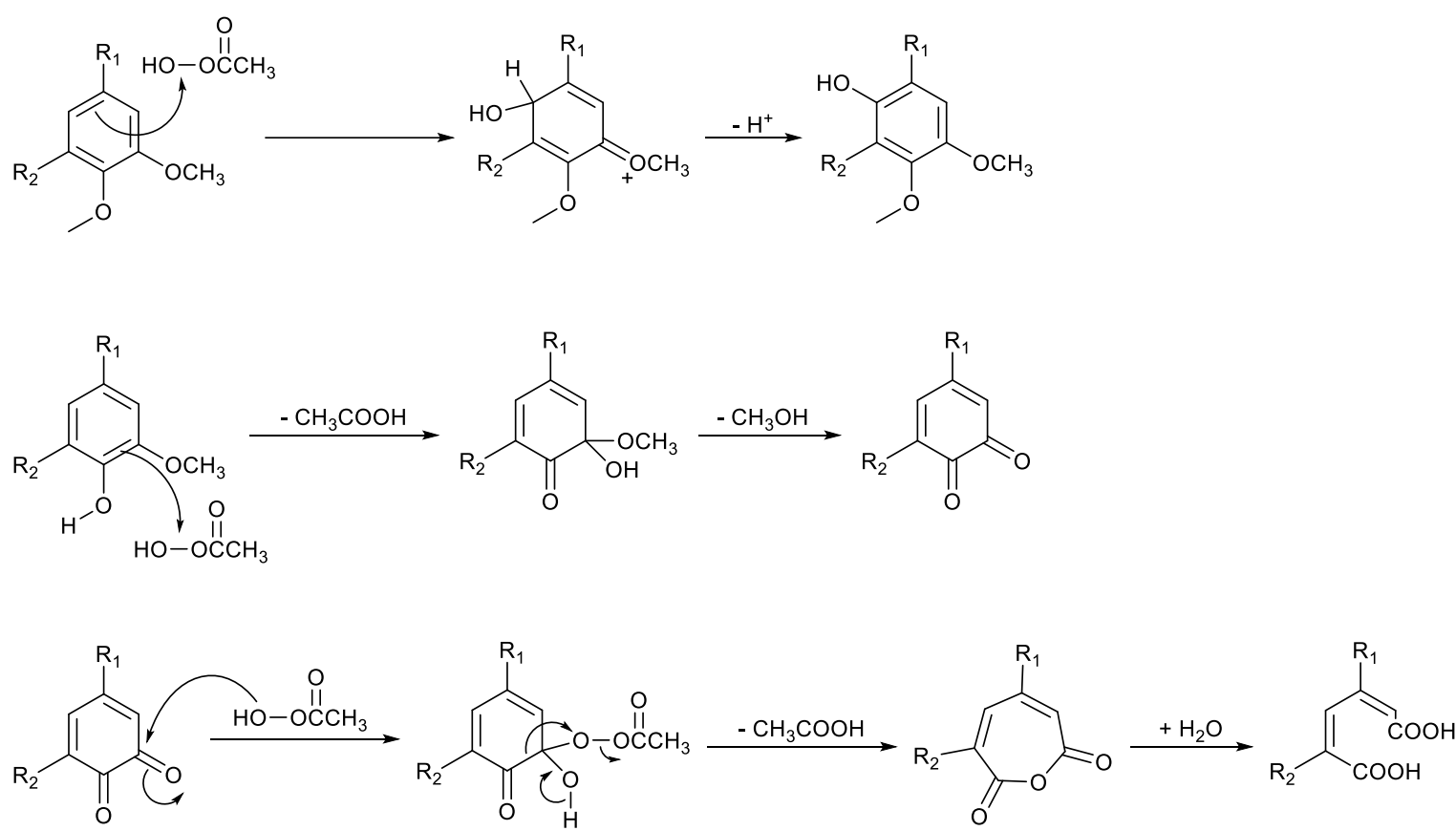

Fig. 1. Mechanisms of hydroxylation and carboxylation of lignin by peracetic acid (Barros et al. 2010)

Plasticizers, such as lignosulfonate (LS) or polycarboxylate ether (PCE), have been used to reduce the amount of added water in cement paste, which is advantageous because 
water reduces the strength of hardened cement (Plank et al. 2010; Huang et al. 2018). LS is a by-product of sulfite pulping, but production of LS has decreased because the sulfite pulping process has been widely displaced by kraft pulping. This LS material increases cement fluidity when it is added into cement paste by using electrostatic repulsion force caused by sulfonate group on its structure. Meanwhile, PCE shows good performance in increasing the fluidity of cement paste. Its structure, called a comb structure, consists of two parts, backbone and side chains. The former contains few carboxyl group to be adsorbed on cement surface, and the latter cause steric hindrance which is the main mechanism to increase cement fluidity for PCE. However, it is expensive to use for this purpose (Cecel et al. 2019), and it would be beneficial to replace this expensive plasticizer.

In this study, simple oxidation of KL by peracetic acid, which was produced by mixing acetic acid and hydrogen peroxide, gave KL a ring-opened chain structure with carboxylate groups by cleaving aromatic rings and produced a water-soluble fraction $(\mathrm{Cx}-$ lig), which was able to act as a plasticizer that disperses cement particles. The cement fluidity caused by the Cx-lig exceeded that caused by commercial LS or PCE. Fouriertransform infrared (FT-IR) spectroscopy, carbon-13 nuclear magnetic resonance $\left({ }^{13} \mathrm{C}\right.$ NMR) spectroscopy, gel permeation chromatography (GPC), elemental analysis, and charge density analysis were used to reveal the structure of the Cx-lig. Based on the analyses, the structure of the $\mathrm{Cx}$-lig was similar to that of polycarboxylates that have many carboxylate groups.

\section{EXPERIMENTAL}

\section{Materials}

Hardwood KL was obtained from Moorim P\&P (Ulsan, Republic of Korea). Acetic acid and hydrogen peroxide, which were purchased from Samchun Chemical Co., Ltd. (Seoul, Republic of Korea), and Daejung Chemicals \& Metals Co., Ltd. (Siheung, Republic of Korea), respectively, were used to make peracetic acid as a reagent. For GPC analysis, pyridine (99.8\%) (Sigma Aldrich, St. Louis, MO, USA) and acetic anhydride $(\geq 99 \%)$ (Sigma Aldrich, St. Louis, MO, USA) were utilized to conduct acetylation. The ${ }^{13} \mathrm{C} \mathrm{NMR}$ analysis was performed with dimethyl sulfoxide-d6 (DMSO-d6, Sigma Aldrich). For measurement of cement fluidity, Portland cement (Asia Cement Co., Ltd, Seoul, Republic of Korea) was purchased. Lignosulfonate and PCE were supplied by Dongnam Co., Ltd. (Pyeongtaek, Republic of Korea).

\section{Methods}

Calculation for Klason lignin and acid-soluble lignin content of KL

The measurement of both Klason lignin and acid-soluble lignin contents of KL was conducted according to NREL/TP-510-42618.

\section{Carboxylation of $K L$}

Peracetic acid was manufactured by mixing acetic acid $(99.5 \%)$ and hydrogen peroxide $(30 \%)$. The mixing ratio (1:4, acetic acid : hydrogen peroxide) was fixed according to the authors' previous research to maximize peracetic acid concentration (Park et al. 2019). A mixture of $1.8 \mathrm{~g}$ of $\mathrm{KL}$ and $13.5 \mathrm{~mL}$ of peracetic acid was reacted at $70{ }^{\circ} \mathrm{C}$ for $80 \mathrm{~min}$. After the reaction, excess water was poured into the reacted solution. Consequently, the lignin-derived compounds that had not completely reacted with the 
peracetic acid precipitated because of the change of $\mathrm{pH}$. Subsequently, the water-soluble and -insoluble fractions were separated by centrifugation. To remove excess acetic acid and hydrogen peroxide, the water-soluble fraction was dialyzed with a CelluSep H1 dialysis tube (Membrane Filtration Products, Inc., St. Seguin, TX, USA) for 24 h, with a change of water every $12 \mathrm{~h}$. Finally, the dialyzed water-soluble fraction was lyophilized to obtain a solid sample.

Comparison of solubility in water of KL and CX-lig

After carboxylation by peracetic acid, to compare the solubility in water, $10 \mathrm{mg}$ of each sample was dissolved in $1 \mathrm{~mL}$ of deionized water at room temperature, separately.

\section{Structural characterization of Cx-lig}

The FT-IR spectra were gathered in the range of $4000 \mathrm{~cm}^{-1}$ to $650 \mathrm{~cm}^{-1}$ with 32 scans at a spectral resolution of $4 \mathrm{~cm}^{-1}$ using a Nicolet 6700 FT-IR spectrometer (Thermo Fisher Scientific, Waltham, MA, USA). The spectrum of the atmosphere was used as a reference. In addition, KL and Cx-lig were analyzed directly on a $\mathrm{ZnSe}$ attenuated total reflectance crystal.

To reveal the changes in functional groups, ${ }^{13} \mathrm{C}$ NMR was utilized. For this analysis, $60 \mathrm{mg}$ of each sample was dissolved in $0.6 \mathrm{~mL}$ of DMSO-d6. The NMR spectra were collected for $8 \mathrm{~h}$ at $60{ }^{\circ} \mathrm{C}$ with a $600 \mathrm{MHz}$ NMR spectrometer (AVANCE 600, Bruker, Billerica, MA, USA)

Before analyzing the molecular weight of the KL, acetylation was performed. For acetylation, $50 \mathrm{mg}$ of each sample was dissolved in $0.5 \mathrm{~mL}$ of pyridine, and $0.5 \mathrm{~mL}$ of acetic anhydride was added. Acetylation was conducted for $2 \mathrm{~h}$ at $105^{\circ} \mathrm{C}$. Next, acetylated KL was obtained by adding the solution into water. Finally, centrifugation and freezedrying were implemented, followed by acetylation. For GPC analysis, $20 \mathrm{mg}$ of solid sample was dissolved in $2 \mathrm{~mL}$ of tetrahydrofuran, which was the hydrophobic mobile phase. Filtration was conducted with a 13JP050AN disposable membrane syringe filter (Advantec, Tokyo, Japan). Number average molecular weight $\left(M_{\mathrm{n}}\right)$, weight average molecular weight $\left(M_{\mathrm{w}}\right)$, and polydispersity index (PDI) were analyzed with a 1260 Infinity II refractive index detector (Agilent Technologies, Santa Clara, CA, USA). The column used was a PLgel $5 \mu \mathrm{m}$ MIXED-C column $(300 \mathrm{~mm} \times 7.5 \mathrm{~mm}$, Agilent Technologies, Santa Clara, CA, USA). The flow rate was $1 \mathrm{~mL} / \mathrm{min}$, and the injection volume was $20 \mu \mathrm{L}$. For the Cx-lig, $20 \mathrm{mg}$ of the sample was dissolved in $2 \mathrm{~mL}$ of deionized water, which was the hydrophilic mobile phase. To remove impurities, a 13HP045AN disposable membrane syringe filter (Advantec, Tokyo, Japan) was utilized. For GPC analysis, a Thermo Dionex HPLC UltiMate 3000 RI system (Thermo Fisher Scientific) was used to measure the $M_{\mathrm{n}}$, $M_{\mathrm{w}}$, and PDI of the Cx-lig. Because the mobile phase was not the same for all GPC analyses, polystyrene and pullulan were utilized as a standard polymer for hydrophobic and hydrophilic samples based on the previous research (Lange et al. 2016) and international standard (ISO/DIS 13885-3), respectively.

To determine the atomic compositions of the KL and Cx-lig, elemental analysis was performed. The elemental content was measured with a Flash EA 1112 (Thermo Electron Co., Waltham, MA, USA). The oxygen content was calculated by subtracting the total fractions of $\mathrm{C}, \mathrm{H}, \mathrm{N}$, and $\mathrm{S}$ from $100 \%$.

Charge density analysis was performed with a streaming current detector (Mütek PCD-03, BTG, Eclépens, Switzerland). Forty milligrams of each sample was dissolved in $40 \mathrm{~mL}$ of deionized water. To obtain the water-soluble fraction, centrifugation was 
conducted with a Mega 17R (Hanil Science Medical, Daejeon, Republic of Korea) at $10,000 \mathrm{rpm}$ for $10 \mathrm{~min}$. Then, $10 \mathrm{~g}$ of Cx-lig solution was used to measure charge density. The standard solution for titration was $1 \mathrm{mmol} / \mathrm{L}$ poly(diallyldimethylammonium chloride). Charge density was calculated using Eq. 1,

$$
q=v c / m
$$

where $q$ is specific charge density (meq/g), $v$ is titrant volume $(\mathrm{mL}), c$ is titrant concentration ( $\mathrm{mol} / \mathrm{L})$, and $m$ is the sample dried weight $(\mathrm{g})$.

To compare cement fluidities after adding plasticizers, a flow table test was conducted according to KS L 5111 (2017). Portland cement (600 g) and plasticizer (0.3 g of Cx-lig, LS, or PCE) were mixed with water $(159.7 \mathrm{~g})$ to make a cement paste. The mixture was mildly mixed for $1 \mathrm{~min}$ and then vigorously stirred for $3 \mathrm{~min}$. Then, the paste was poured into a flow table mold, and the table was dropped 25 times to flatten the paste after removing the mold. Finally, the diameter of cement paste was measured in three directions through the center of the cement paste.

\section{RESULTS AND DISCUSSION}

The Cx-lig, in which carboxylate groups were introduced in the KL, was prepared by peracetic acid oxidation, as shown in Fig. 2. The KL was not soluble in deionized water, but the Cx-lig could be completely dissolved in water. The Cx-lig was subsequently analyzed to reveal its chemical structure.

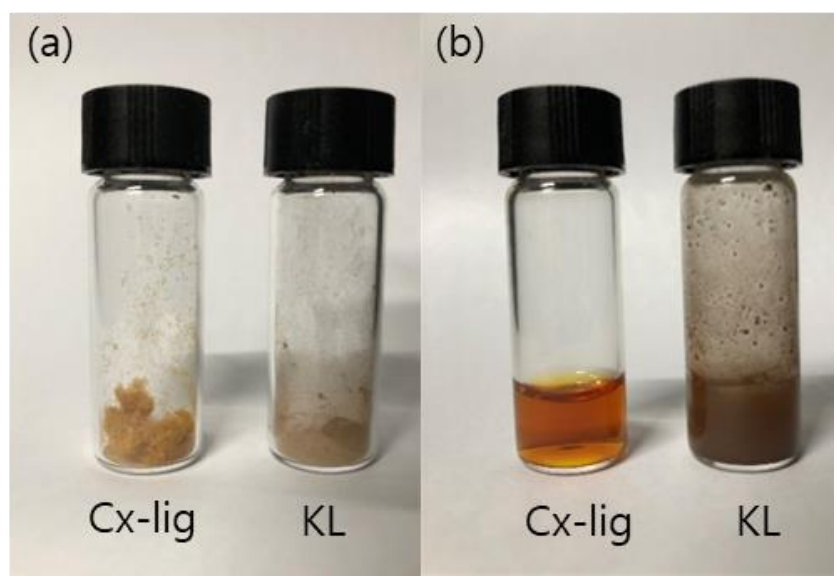

Fig. 2. Physical appearance of $K L$ and $C x$-lig (a) before and (b) after dissolving in water

\section{Klason Lignin and Acid-soluble Lignin Content of KL}

To measure the pure lignin content of KL, Klason lignin and acid-soluble lignin contents were calculated (Table 1).

Table 1. Klason Lignin and Acid Soluble Lignin Content of KL

\begin{tabular}{|c|c|c|c|}
\hline & Klason lignin & Acid-soluble lignin & Total \\
\hline Contents & $90.26 \%( \pm 0.51)$ & $8.60 \%( \pm 0.53)$ & $98.86( \pm 0.10)$ \\
\hline
\end{tabular}




\section{Chemical Structural Analysis of Cx-lig}

Changes in the FT-IR spectra of the KL and Cx-lig were observed (Fig. 3). Before carboxylation by peracetic acid, the KL showed peaks for methoxy groups at $2915 \mathrm{~cm}^{-1}$ and $2848 \mathrm{~cm}^{-1}$ and aromatic ring units from $1620 \mathrm{~cm}^{-1}$ to $1400 \mathrm{~cm}^{-1}$ (Coates 2006). However, after carboxylation, the peaks for the aromatic units and the methoxy groups became weaker because the peracetic acid introduced carboxylate groups by reacting with phenolic hydroxyl and methoxy groups, according to the mechanism shown in Fig. 1. In the same manner, the guaiacyl ring absorption peaks at $1322 \mathrm{~cm}^{-1}$ and $1267 \mathrm{~cm}^{-1}$ became weaker after carboxylation. The former was assigned to the condensed structure of both syringyl and guaiacyl units, and the latter was for guaiacyl units (Boeriu et al. 2004). This result showed that the peracetic acid reacted with the syringyl and guaiacyl units, resulting in ring cleavage. Additionally, peaks for the carboxylate groups appeared at $1715 \mathrm{~cm}^{-1}$, due to $\mathrm{C}=\mathrm{O}$ stretching, and $1172 \mathrm{~cm}^{-1}$, due to $\mathrm{C}-\mathrm{O}$ stretching, for the $\mathrm{Cx}$-lig structure (Chen et al. 2015). After carboxylation, the intensities of these two peaks exceeded those of the KL. The increases of these two peaks indicated that the phenolic hydroxyl and methoxy groups were successfully oxidized, leading to the introduction of the carboxylate groups during the peracetic acid treatment. Also, the authors' previous research revealed by FT-IR spectroscopy that using peracetic acid is one of the ways to produce lignin derivatives with carboxylate group (Park et al. 2020).

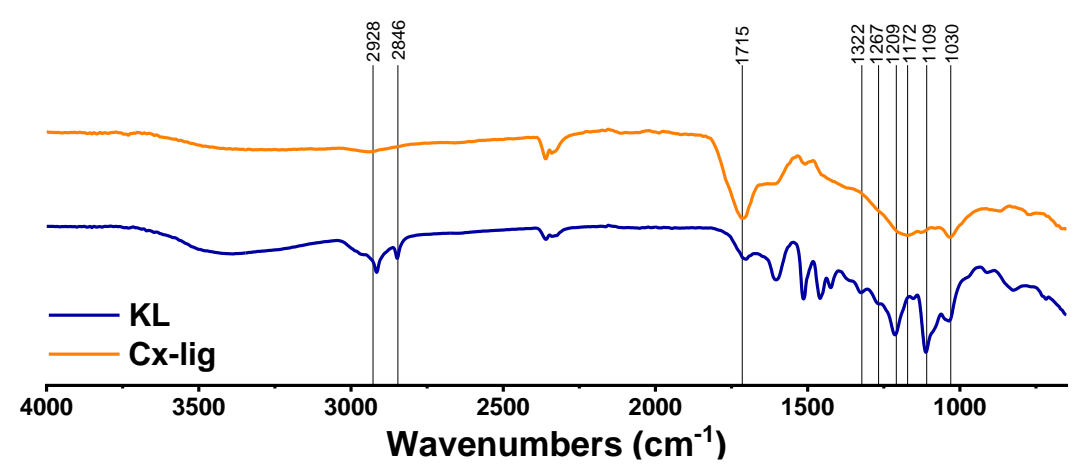

Fig. 3. FT-IR spectra of $\mathrm{KL}$ and $\mathrm{Cx}-\mathrm{lig}$

Figure 4 shows the ${ }^{13} \mathrm{C}$ NMR spectra of the KL and Cx-lig. The peaks from 160 ppm to $180 \mathrm{ppm}$ were assigned to the carboxylate group carbon. For the $\mathrm{Cx}-\mathrm{lig}$, there were broad peaks in this range because each carboxylate group was in a different chemical environment in the structure. By its nature, $\mathrm{KL}$ is a heterogeneous polymer, so the carbon atoms are in a variety of chemical environments (Gellerstedt and Robert 1987). For this reason, carbons of each carboxylate group were shielded differently after carboxylation, such that broad peaks appeared for the carboxylate groups in the NMR spectra of the Cxlig. Furthermore, the peak at $126 \mathrm{ppm}$, which was assigned to the $\mathrm{C}=\mathrm{C}$ bond, appeared after carboxylation by peracetic acid. This result implied that the final product had two double bonds on its structure, according to the previously mentioned reaction mechanism (Fig. 1). Moreover, the peaks near $148 \mathrm{ppm}$ and $56 \mathrm{ppm}$ were assigned to the aromatic ring and methoxy group carbons, respectively ( $\mathrm{Lu}$ et al. 2017). The intensities of these peaks decreased due to the ring cleavage reaction by the peracetic acid, according to the reaction mechanism. 


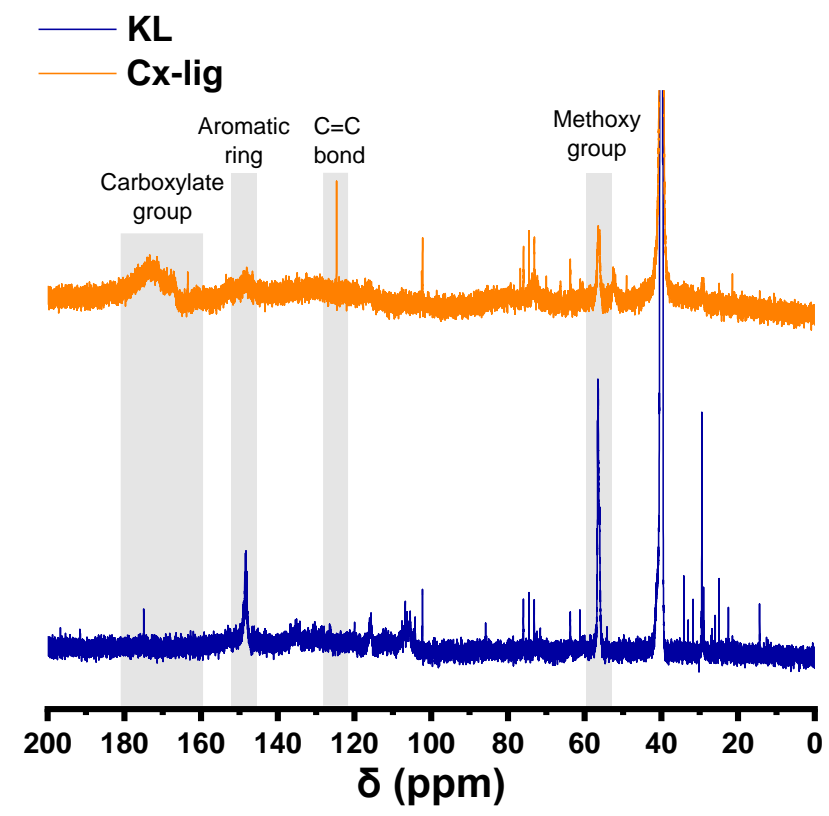

Fig. 4. ${ }^{13} \mathrm{C}$ NMR spectra of $\mathrm{KL}$ and $\mathrm{Cx}$-lig

To reveal whether carboxylate groups were introduced in the Cx-lig, elemental analysis was performed. Figure 5 presents the elemental compositions of the KL and Cxlig. In the KL, the contents of $\mathrm{C}, \mathrm{O}, \mathrm{H}, \mathrm{N}$, and $\mathrm{S}$ were $59.51 \%, 31.70 \%, 5.87 \%, 0.01 \%$, and $2.91 \%$, respectively. In contrast, the elemental ratios in Cx-lig for each atom were $49.78 \%$, $45.26 \%, 4.19 \%, 0.13 \%$, and $1.25 \%$, respectively. After carboxylation of the KL by the peracetic acid, the oxygen content increased, indicating successful carboxylation by the peracetic acid. Similar steps produced carboxymethylated hardwood KL. After carboxymethylation to attach the carboxylate groups onto KL, the oxygen content increased (Konduri et al. 2015). Moreover, sulfur content decreased, indicating that the elimination reaction of thiol also occurred during peracetic acid oxidation.

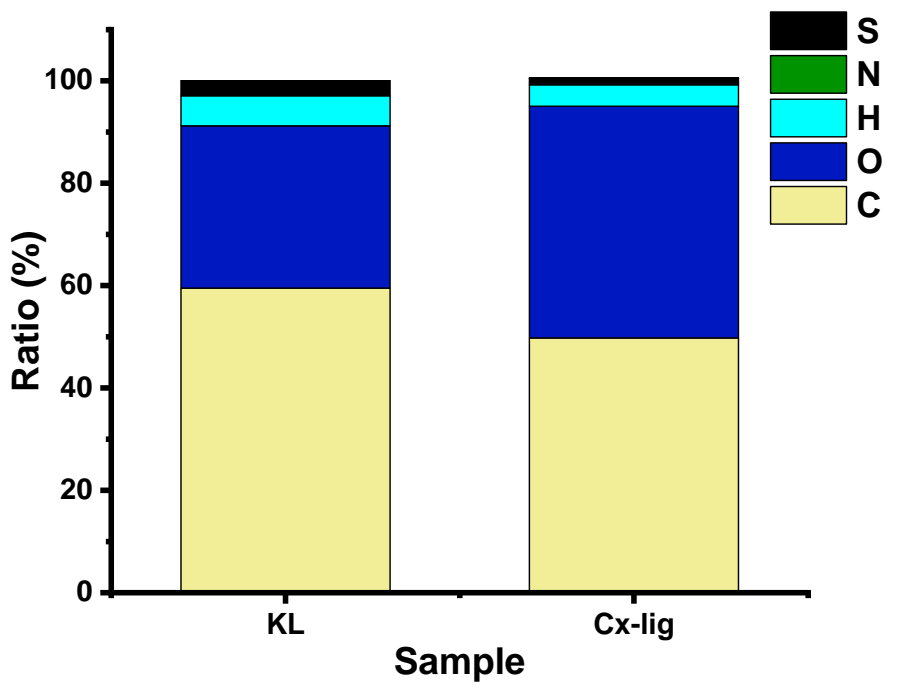

Fig. 5. Elemental compositions of the $\mathrm{KL}$ and $\mathrm{Cx}$-lig 
The GPC-determined values of the $M_{\mathrm{n}}, M_{\mathrm{w}}$, and PDI of the KL, Cx-lig (nondialysis), and Cx-lig (dialysis) are shown in Table 1. Carboxylation caused the PDI to decrease substantially. The $M_{\mathrm{n}}$ of the KL was less than that of the Cx-lig (non-dialysis). It has been reported that hydrogen peroxide is able to divide into two hydroxyl radicals by a metal ion (Zhou and Lu 2014). These radicals attack lignin molecules and produce lignin radicals, which have a high reactivity to collide into one another. When lignin radicals collide with each other, new bonds, such as $\beta-\beta$ and $\beta-5$, are produced. Consequently, $M_{\mathrm{n}}$ increases due to the condensation reaction (Kim and Kim 2018). Additionally, Cx-lig (dialysis) had a greater $M_{\mathrm{w}}$ than Cx-lig (non-dialysis). This result means that $M_{\mathrm{w}}$ was able to increase by dialysis, which removes low-molecular-weight molecules.

Table 2. $M_{\mathrm{n}}, M_{\mathrm{w}}$, and PDI of $\mathrm{KL}, \mathrm{Cx}-$ lig (non-dialysis), and $\mathrm{Cx}$-lig (dialysis)

\begin{tabular}{|c|c|c|c|}
\hline & $\mathrm{KL}$ & $\begin{array}{c}\text { Cx-lig } \\
\text { (non-dialysis) }\end{array}$ & $\begin{array}{c}\text { Cx-lig } \\
\text { (dialysis) }\end{array}$ \\
\hline$M_{\mathrm{n}}(\mathrm{Da})$ & 1242 & 1634 & 2898 \\
\hline$M_{\mathrm{w}}(\mathrm{Da})$ & 3849 & 2303 & 4811 \\
\hline $\mathrm{PDI}$ & 3.10 & 1.41 & 1.66 \\
\hline
\end{tabular}

\section{Charge Density Analysis of Cx-lig}

To anticipate the performances of the plasticizers, charge densities were compared. The charge density values for the KL, PCE, LS, and Cx-lig are shown in Fig. 6. Charge density is one of the key factors to increasing cement fluidity (He and Fatehi 2015). When the material, which was negatively charged, was added to the cement paste, it was adsorbed onto the surfaces of the cement particles. This negative charge caused a repulsive force among the cement particles. Consequently, negatively charged materials were able to increase the fluidity of cement paste (Pérez-Nicolás et al. 2016). The measured charge density values of the KL, LS, and PCE were $0.00 \mathrm{meq} / \mathrm{g},-1.19 \mathrm{meq} / \mathrm{g}$, and $-0.08 \mathrm{meq} / \mathrm{g}$, respectively. Other research has shown similar results for the charge densities of LS (Oveissi and Fatehi 2015; Aro and Fatehi 2017) and PCE (Plank et al. 2009). Meanwhile, the charge density of the Cx-lig was $-1.94 \mathrm{meq} / \mathrm{g}$, which was greater than the values for LS and PCE.

Kraft lignin contains few functional groups that indicate negative or positive charge (Sjöström 1993), and its charge density was effectively zero. LS is a by-product of sulfite pulping, and it has a sulfonate group, making it negatively charged. Based on the structure of LS, the sulfonate group is heterogeneously attached to the aliphatic chain region (Lange et al. 2013). For this reason, the LS had a fairly high charge density. Compared to the LS, the PCE had a much lower charge density. This result was because PCE consists of two parts, a negatively charged part and a non-charged chain structure part (Qian and De Schutter 2018). The low charge density of PCE means that there is a small amount of the negatively charged part, which is needed for the PCE to adsorb onto the surface of the cement. After PCE is adsorbed onto the surface of the cement particle, a non-charged chain structure causes steric hindrance among the cement particles (Plank et al. 2010). For these reasons, the charge density of PCE does not need to be high. In the Cx-lig, a number of carboxylate groups were introduced by the peracetic acid because KL is mainly composed of an aromatic ring structure. As a consequence of the added carboxylate groups, the measured charge density was high. 


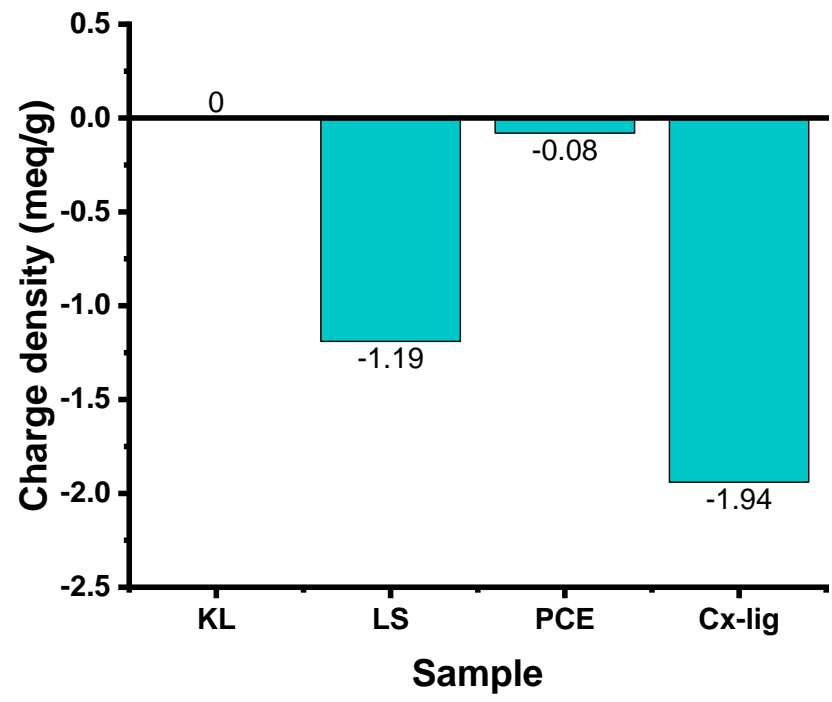

Fig. 6. Charge densities of KL, LS, PCE, and Cx-lig

\section{Cement Fluidity Analysis}

To characterize the performances of the plasticizers, mortar table tests were conducted. Figure 7 presents a comparison of the cement paste diameter when each plasticizer was added. The diameter of the cement paste without any plasticizer was 177.00 $\mathrm{mm} \pm 1.73 \mathrm{~mm}$. When each commercial plasticizer, LS and PCE, was added into the cement paste, the diameters were $189.33 \mathrm{~mm} \pm 4.16 \mathrm{~mm}$ and $199.67 \mathrm{~mm} \pm 1.53 \mathrm{~mm}$, respectively. Compared to the commercial plasticizers, use of $\mathrm{Cx}$-lig resulted in a greater cement paste diameter of $206.11 \mathrm{~mm} \pm 1.36 \mathrm{~mm}$.

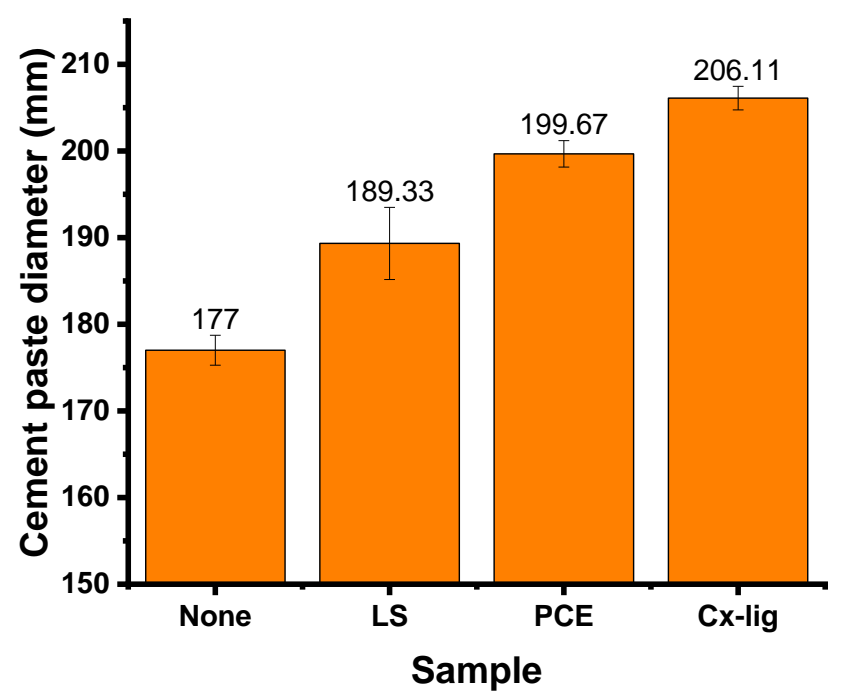

Fig. 7. Cement paste diameter after adding LS, PCE, and Cx-lig

The increased cement fluidity with PCE was mainly due to its branched structure and steric hindrance. In contrast, LS chiefly affected cement fluidity by electrostatic repulsion (Qian and De Schutter 2018). The Cx-lig had carboxylate groups in its structure, 
so the functional groups may have increased the cement fluidity in the manner of LS, rather than that of PCE. Many studies have revealed that PCE performs well as a plasticizer to increase cement fluidity due to a non-charged comb structure (Shin et al. 2008; Lei and Plank 2014; Chuang et al. 2019). In this experiment, adding Cx-lig, which exhibited greater charge density, was able to increase the workability of the cement paste. In conclusion, this result demonstrates that increasing charge density can be one way to increase the fluidity of cement.

\section{CONCLUSIONS}

1. Polycarboxylates based on kraft lignin (KL) were able to substitute for commercial plasticizers. Kraft lignin could be modified using peracetic acid, which cleaves aromatic rings, resulting in the introduction of carboxylate groups, to produce polycarboxylates for a plasticizer.

2. Because of the carboxylate groups, Cx-lig exhibited a greater charge density than lignosulfonate (LS) and polycarboxylate ether (PCE). Furthermore, Cx-lig provided greater cement workability than LS and PCE when added to cement paste.

3. Charge density was as important a factor as a comb structure to increasing cement workability.

\section{ACKNOWLEDGMENTS}

This study was carried out with the support of ' $R \& D$ Program for Forest Science Technology (2019158A00-1920-0001, 2020215C10-2022-AC01)' provided by Korea Forest Service (Korea Forestry Promotion Institute). Kraft lignin and commercial plasticizers were kindly supplied by Moorim P\&P (Ulsan, Republic of Korea) and Dongnam Co., Ltd. (Pyeongtaek, Republic of Korea), respectively.

\section{REFERENCES CITED}

Aro, T., and Fatehi, P. (2017). "Production and application of lignosulfonates and sulfonated lignin," ChemSusChem 10(9), 1861-1877. DOI: 10.1002/cssc.201700082

Barros, D. P., Silva, V. L., Hämäläinen, H., and Colodette, J. L. (2010). "Effect of last stage bleaching with peracetic acid on brightness development and properties of eucalyptus pulp,” BioResources 5(2), 881-898. DOI: 10.15376/biores.5.2.881-898

Boeriu, C. G., Bravo, D., Gosselink, R. J. A., and van Dam, J. E. G. (2004). "Characterisation of structure-dependent functional properties of lignin with infrared spectroscopy," Industrial Crops and Products 20(2), 205-218. DOI: 10.1016/j.indcrop.2004.04.022

Cecel, R. T., Abrão, P. C. R. A., Cardoso, F. A., and John, V. M. (2019). "Consumption of superplasticizer admixture for different cements and their binder efficiency," Revista IBRACON de Estruturas e Materiais 12(6), 1260-1287. DOI: 10.1590/s198341952019000600003 
Chakar, F. S., and Ragauskas, A. J. (2004). "Review of current and future softwood kraft lignin process chemistry," Industrial Crops and Products 20(2), 131-141. DOI: 10.1016/j.indcrop.2004.04.016

Chen, Y., Zou, C., Mastalerz, M., Hu, S., Gasaway, C., and Tao, X. (2015). “Applications of micro-Fourier transform infrared spectroscopy (FTIR) in the geological sciencesA review," International Journal of Molecular Sciences 16(12), 30223-30250. DOI: 10.3390/ijms 161226227

Chuang, P.-H., Tseng, Y.-H., Fang, Y., Gui, M., Ma, X., and Luo, J. (2019). "Effect of side chain length on polycarboxylate superplasticizer in aqueous solution: A computational study," Polymers 11(2). DOI: 10.3390/polym11020346

Coates, J. (2006). "Interpretation of infrared spectra, a practical approach," in: Encyclopedia of Analytical Chemistry: Applications, Theory and Instrumentation, R. A. Meyers (ed.), John Wiley \& Sons, Hoboken, NJ, USA, pp. 10815-10837. DOI: 10.1002/9780470027318.a5606

El Mansouri, N.-E., Yuan, Q., and Huang, F. (2011). "Synthesis and characterization of kraft lignin-based epoxy resins," BioResources 6(3), 2492-2503.

Ferdosian, F., Yuan, Z., Anderson, M., and Xu, C. C. (2012). "Chemically modified lignin through epoxidation and its thermal properties," Journal of Science \& Technology for Forest Products and Processes 2(4), 11-15.

Figueiredo, P., Ferro, C., Kemell, M., Liu, Z., Kiriazis, A., Lintinen, K., Florindo, H. F., Yli-Kauhaluoma, J., Hirvonen, J., Kostiainen, M. A., and Santos, H. A. (2017). "Functionalization of carboxylated lignin nanoparticles for targeted and $\mathrm{pH}-$ responsive delivery of anticancer drugs," Nanomedicine 12(21), 2581-2596. DOI: 10.2217/nnm-2017-0219

Gao, W., Inwood, J. P. W., and Fatehi, P. (2019). "Sulfonation of phenolated kraft lignin to produce water soluble products," Journal of Wood Chemistry and Technology 39(4), 225-241. DOI: 10.1080/02773813.2019.1565866

Gellerstedt, G., and Robert, D. (1987). "Quantitative ${ }^{13} \mathrm{C}$ NMR analysis of kraft lignins," Acta Chemica Scandinavica B41, 541-546. DOI: 10.3891/acta.chem.scand.41b-0541

He, W., and Fatehi, P. (2015). "Preparation of sulfomethylated softwood kraft lignin as a dispersant for cement admixture," RSC Advances 5(58), 47031-47039. DOI: $10.1039 / \mathrm{c} 5 \mathrm{ra0} 4526 \mathrm{f}$

Huang, C., Ma, J., Zhang, W., Huang, G., and Yong, Q. (2018). "Preparation of lignosulfonates from biorefinery lignins by sulfomethylation and their application as a water reducer for concrete," Polymers 10(8). DOI: 10.3390/polym10080841

ISO/DIS 13885-3 (2020). "Gel permeation chromatography (GPC) - Part 3: Water as eluent," International Organization for Standardization, Geneva, Switzerland.

Kim, K. H., and Kim, C. S. (2018). "Recent efforts to prevent undesirable reactions from fractionation to depolymerization of lignin: Toward maximizing the value from lignin," Frontiers in Energy Research 6. DOI: 10.3389/fenrg.2018.00092

Konduri, M. K., Kong, F., and Fatehi, P. (2015). "Production of carboxymethylated lignin and its application as a dispersant," European Polymer Journal 70, 371-383. DOI: 10.1016/j.eurpolymj.2015.07.028

KS L 5111 (2017). "Flow table for use in tests of hydraulic cement," Korean Standards Association, Seoul, Republic of Korea.

Lange, H., Decina, S., and Crestini, C. (2013). "Oxidative upgrade of lignin - Recent routes reviewed," European Polymer Journal 49(6), 1151-1173. DOI: 10.1016/j.eurpolymj.2013.03.002 
Lange, H., Rulli, F., and Crestini, C. (2016). "Gel permeation chromatography in determining molecular weights of lignins: Critical aspects revisited for improved utility in the development of novel materials," ASC Sustainable Chemistry \& Engineering 4, 5167-5180. DOI: 10.1021/acssuschemeng.6b00929

Lee, M. Y., and Park, W. H. (2000). "Preparation of bacterial copolyesters with improved hydrophilicity by carboxylation," Macromolecular Chemistry and Physics 201(18), 2771-2774. DOI: 10.1002/1521-3935(20001201)201:18<2771::AIDMACP2771>3.0.CO;2-V

Lei, L., and Plank, J. (2014). "Synthesis and properties of a vinyl ether-based polycarboxylate superplasticizer for concrete possessing clay tolerance," Industrial \& Engineering Chemistry Research 53(3), 1048-1055. DOI: 10.1021/ie4035913

Lu, Y., Lu, Y.-C., Hu, H.-Q., Xie, F.-J., Wei, X.-Y., and Fan, X. (2017). "Structural characterization of lignin and its degradation products with spectroscopic methods," Journal of Spectroscopy 2017. DOI: 10.1155/2017/8951658

Luo, Y.-R. (2012). "Bond dissociation energies," in: CRC Handbook of Chemistry and Physics, W. M. Haynes, D. R. Lide, and T. J. Bruno (eds.), CRC Press, Boca Raton, FL, USA, pp. 9-65 - 9-96.

Narani, A., Chowdari, R. K., Cannilla, C., Bonura, G., Frusteri, F., Heeres, H. J., and Barta, K. (2015). "Efficient catalytic hydrotreatment of kraft lignin to alkylphenolics using supported NiW and NiMo catalysts in supercritical methanol," Green Chemistry 17(11), 5046-5057. DOI: 10.1039/c5gc01643f

Ouyang, X., Ke, L., Qiu, X., Guo, Y., and Pang, Y. (2009). "Sulfonation of alkali lignin and its potential use in dispersant for cement," Journal of Dispersion Science and Technology 30(1), 1-6. DOI: 10.1080/01932690802473560

Oveissi, F., and Fatehi, P. (2015). "Characterization of four different lignins as a first step toward the identification of suitable end-use applications," Journal of Applied Polymer Science 132(32). DOI: 10.1002/app.42336

Park, S.-Y., Cho, S.-M., Kim, J.-C., Hong, C., Kim, S.-H., Ryu, G.-H., and Choi, I.-G. (2019). "Effects of peracetic acid and hydrogen peroxide concentration on kraft lignin degradation at room temperature," BioResources 14(2), 4413-4429. DOI: 10.15376/biores. 14.2.4413-4429

Park, S.-Y., Choi, J. -H., Kim, J.-H., Cho, S.-M., Yeon, S., Jeong, H., Lee, S. M., and Choi, I.-G. (2020). "Peracetic acid-induced kraft lignin solubilization and its characterization for selective production of macromolecular biopolymers," International Journal of Biological Macromolecules in press. DOI: 10.1016/j.ijbiomac.2020.06.041

Pérez-Nicolás, M., Duran, A., Navarro-Blasco, I., Fernández, J. M., Sirera, R., and Alvarez, S. J. I. (2016). "Study on the effectiveness of PNS and LS superplasticizers in air lime-based mortars," Cement and Concrete Research 82, 11-22. DOI: 10.1016/j.cemconres.2015.12.006

Plank, J., Schroefl, C., Gruber, M., Lesti, M., and Sieber, R. (2009). "Effectiveness of polycarboxylate superplasticizers in ultra-high strength concrete: The importance of PCE compatibility with silica fume," Journal of Advanced Concrete Technology 7(1), 5-12. DOI: $10.3151 /$ jact.7.5

Plank, J., Zhimin, D., Keller, H., Hössle, F. v., and Seidl, W. (2010). "Fundamental mechanisms for polycarboxylate intercalation into $\mathrm{C}_{3} \mathrm{~A}$ hydrate phases and the role of sulfate present in cement," Cement and Concrete Research 40(1), 45-57. DOI: 10.1016/j.cemconres.2009.08.013 
Qian, Y., and De Schutter, G. (2018). "Different effects of NSF and PCE superplasticizer on adsorption, dynamic yield stress and thixotropy of cement pastes," Materials 11(5). DOI: 10.3390/ma11050695

Shin, J.-Y., Hong, J.-S., Suh, J.-K., and Lee, Y.-S. (2008). "Effects of polycarboxylatetype superplasticizer on fluidity and hydration behavior of cement paste," Korean Journal of Chemical Engineering 25(6), 1553-1561. DOI:10.1007/s11814-008-0255-3

Sjöström, E. (1993). Wood Chemistry: Fundamentals and Applications, $2^{\text {nd }}$ Edition, Academic Press, Inc., San Diego, CA, USA. DOI: 10.1016/C2009-0-03289-9.

Sun, J., Wang, C., Stubbs, L. P., and He, C. (2017). "Carboxylated lignin as an effective cohardener for enhancing strength and toughness of epoxy," Macromolecular Materials and Engineering 302(12). DOI: 10.1002/mame.201700341

Zhao, G., He, C., Yin, P., Imler, G. H., Parrish, D. A., and Shreeve, J. M. (2018). "Efficient construction of energetic materials via nonmetallic catalytic carbon-carbon cleavage/oxime-release-coupling reactions," Journal of the American Chemical Society 140(10), 3560-3563. DOI: 10.1021/jacs.8b01260

Zheng, T., Zheng, D., Qiu, X., Yang, D., Fan, L., and Zheng, J. (2019). “A novel branched claw-shape lignin-based polycarboxylate superplasticizer: Preparation, performance and mechanism," Cement and Concrete Research 119, 89-101. DOI: 10.1016/j.cemconres.2019.03.007

Zhou, X.-F., and Lu, X.-J. (2014). "Structural characterization of kraft lignin for its green utilization," Wood Research 59(4), 583-592.

Article submitted: June 8, 2020; Peer review completed: August 3, 2020; Revised version received: September 3, 2020; Accepted: September 4, 2020; Published: September 9, 2020.

DOI: $10.15376 /$ biores.15.4.8133-8145 